\title{
Expression of Notch receptors and their ligands in pancreatic ductal adenocarcinoma
}

\author{
HAI-YAN SONG ${ }^{1,2}$, YING WANG ${ }^{2}$, HONG LAN $^{2}$ and YU-XIANG ZHANG ${ }^{2}$ \\ ${ }^{1}$ School of Basic Medical Sciences, Xinxiang Medical University, Henan Collaborative \\ Innovation Center of Molecular Diagnosis and Laboratory Medicine, Xinxiang, Henan 453003; \\ ${ }^{2}$ Department of Biochemistry and Molecular Biology, Capital Medical University, Beijing 100069, P.R. China
}

Received June 22, 2017; Accepted January 4, 2018

DOI: $10.3892 / \mathrm{etm} .2018 .6172$

\begin{abstract}
Pancreatic cancer is the fourth leading cause of cancer-associated mortality in developed countries. Pancreatic ductal adenocarcinoma (PDAC) accounts for $\sim 90 \%$ of all pancreatic cancer cases. The Notch signaling pathway serves a crucial role in embryonic development, as well as during the tumorigenesis of different types of cancer. However, Notch signaling serves either oncogenic or tumor suppressor roles depending on the tissue type. There are four Notch receptors (Notch1-4) and five ligands [Jagged1, Jagged2, $\delta$-like ligand protein (DLL)1, DLL3 and DLL4]; therefore, it has been suggested that the different Notch receptors serve distinct roles in the same type of tissue. To determine whether this is the case, the present study measured the expression of all Notch receptors and their ligands in PDAC tissue samples and cells. Immunohistochemistry was performed to measure the expression of Notch receptors and their ligands in paraffin-embedded PDAC tissue samples. Immunofluorescence was used to detect the expression of Notch receptors in the pancreatic cancer cell lines human pancreatic adenocarcinoma (HPAC) and PANC-1. In addition, levels of Notch receptors and ligands in HPAC and PANC-1 cells were analyzed by western blot analysis. The results revealed that levels of Notch1 and Notch3 were increased in PDAC tissues, whereas levels of Notch2 and Notch3 were not. The expression of Notch receptors in the pancreatic cancer cell lines HPAC and PANC-1 was consistent with their expression in PDAC tissues. Additionally, levels of the ligands DLL1, DLL3 and DLL4 were increased in HPAC and PANC-1 cells, as well as PDAC tissue samples. However, the expression of Jagged1 and 2 remained low. These results indicate that Notch1, Notch3, DLL1, DLL3 and DLL4 are upregulated in PDAC, a positive correlation was observed
\end{abstract}

Correspondence to: Professor Yu-Xiang Zhang, Department of Biochemistry and Molecular Biology, Capital Medical University, 10 Xitoutiao, Beijing 100069, P.R. China

E-mail: zhouyike2009@126.com

Key words: Notch receptors, Notch ligands, pancreatic cancer, expression, cancer biomarkers between the expression of Notch1 and Notch3, and between Notch1 and the ligands DLL1, DLL3 and DLL4. whereas Notch2, Notch4, Jagged1 and Jagged2 are not. The interaction of Notch1 and Notch3 with Notch ligands DLL1, DLL3 and DLL4 may be important in maintaining the tumor phenotype of pancreatic cancer.

\section{Introduction}

Pancreatic ductal adenocarcinoma (PDAC) accounts for $>90 \%$ of all pancreatic cancer cases (1). It represents a severe health risk and is the fourth leading cause of cancer-associated mortality in developed countries (2). PDAC is a highly aggressive malignancy with a poor prognosis; the five-year survival rate is $<5 \%$ (3). As PDAC is often diagnosed at an advanced stage with metastasis, it is often too late for patients to undergo curative surgery and traditional chemotherapy is not an effective treatment strategy (4). Therefore, it is critical to understand the molecular mechanisms underlying the development and progression of PDAC to enable the development of novel strategies to inhibit tumor development, impede tumor growth and reduce the recurrence rate of the disease.

In mammals, Notch is a highly conserved gene family that includes the Notch1-4 receptors and their ligands: $\delta$-like ligand protein (DLL)1, DLL3, DLL4, Jagged1 and Jagged2 (5). The Notch signaling pathway consists of Notch receptors, their ligands and C-promoter binding factor 1, suppressor of hairless, Lag-1 (CSL), DNA binding proteins and downstream target genes that are involved in regulating cell functions, including proliferation, differentiation and apoptosis (6,7). Binding of the Notch receptor to its ligand in adjacent cells activates the Notch signaling pathway (8). Notch receptor proteins are sheared by proteolytic enzymes, releasing the C-terminal intracellular domain (NICD), which then translocates into the nucleus. In the nucleus, the NICD binds to CSL, changing CSL from a transcriptional repressor to an activator (9). This leads to the activation of downstream Notch target genes, including Hes and Hey family genes (5). The Notch signaling pathway regulates pancreatic cell differentiation in the developing pancreas (6) and participates in the development and progression of PDAC (10-13). Although previous studies have described the activation of Notch signaling components in PDAC (14-17), the link between elevated Notch expression and tumorigenesis 
in PDAC is controversial as contradictory results have been reported by different studies. Mazur et al (11) demonstrated that Notch signaling has a tumor promoting effect, whereas Hanlon et al (18) demonstrated that it had an inhibitory tumor effect $(11,18)$. In addition, the expression pattern of the Notch receptors and ligands in PDAC remains unclear.

The high expression of a potential oncogene means that it serves a significant role in cancer (17). Therefore, to elucidate the role of Notch signaling in PDAC, in the current study, immunohistochemical staining was performed on samples collected from 24 patients with the necessary associated clinical data. Immunofluorescence staining and western blot analysis were also performed to detect the expression of Notch receptors and their ligands in the pancreatic cancer human pancreatic adenocarcinoma (HPAC) and PANC-1 cell lines.

\section{Materials and methods}

Cell lines. The PDAC cell lines HPAC and PANC-1 and the $293 \mathrm{~T}$ cells and HeLa cell lines were all purchased from the cell bank of the Chinese Academy of Sciences (Beijing, China). PANC-1 and 293 cells were maintained in Dulbecco's modified Eagle's medium (DMEM)-high glucose (Hyclone ${ }^{\mathrm{TM}}$; GE Healthcare Life Sciences, Logan, UT, USA) supplemented with $10 \%$ fetal bovine serum (FBS; Zhejiang Tianhang Biotechnology Co., Ltd., Huzhou, China), $1 \%$ penicillin and $1 \%$ streptomycin (Beyotime Institute of Biotechnology, Haimen, China). HPAC cells and HeLa cells were maintained in RPMI-1640 (GE Healthcare Life Sciences) supplemented with $10 \%$ FBS, $1 \%$ penicillin and $1 \%$ streptomycin. All cells were maintained at $37^{\circ} \mathrm{C}$ in $5 \% \mathrm{CO}_{2}$.

A total of 24 PDAC tissues were collected from patients who underwent surgery for pancreatic cancer at the Affiliated Center Hospital of Xinxiang Medical University (Xinxiang, China) from May 2010 to July 2015. PDAC tissues were then formalin-fixed ( $10 \%$ formalin for $24 \mathrm{~h}$ at room temperature) and paraffin-embedded. The study protocol adhered to The Code of Ethics of the World Medical Association (Declaration of Helsinki). The present study was approved by the Ethics Committee of the Affiliated Center Hospital of Xinxiang Medical University. Written informed consent was obtained from all patients prior to the procedure. Patient information is listed in Table I. PDAC tissues were confirmed using histopathological analysis.

Immunohistochemistry. For histological assessment, immunohistochemical analysis was performed using 5- $\mu$ m-thick PDAC tissue sections. Xylene and graded alcohols were used for dewaxing and rehydration. Subsequently, sections were treated with citrate salt buffer $(\mathrm{pH}$ 6.0) in a microwave for $15 \mathrm{~min}$ for antigen retrieval $\left(100^{\circ} \mathrm{C}\right)$, followed by incubation with $3 \%$ hydrogen peroxide for 15 min to block endogenous peroxidase activity at room temperature. The samples were blocked with 5\% donkey blood serum (Jackson ImmunoResearch Laboratories, Inc., West Grove, PA, USA) in PBS for $1 \mathrm{~h}$ at room temperature. The primary antibodies used in the experiments are listed in Table II. Samples were incubated with the primary antibodies at $4^{\circ} \mathrm{C}$ overnight, followed by incubation with secondary horseradish peroxidase (HRP)-conjugated antibodies (cat. no. SP-9001; OriGene
Technologies, Inc., Beijing, China) for $1 \mathrm{~h}$ at room temperature. Diaminobenzidine and hematoxylin were used for staining (20 sec) and counterstaining (10 sec), respectively at room temperature. Following dehydration with graded alcohols and xylene, slides were sealed with coverslips and neutral gum. The negative control group was incubated with PBS instead of the primary antibody. Staining intensities were quantified by two pathologists blinded to the sample group. The Video Pro32 color image analysis system was used, using the Grey value and optical density value to analyze the immunohistochemical positive expression strength. The intensity of Notch receptors and ligands staining was scored using the following scoring system: 0 (no appreciable staining; negative), 1 (barely detectable staining; weak positive), 2 (readily identifiable brown staining; positive) and 3 (dark brown staining; strong positive). The total score was calculated by multiplying the percentage of positive cells and the intensity score. A tumor sample was considered positive if the score was $\geq 4$ and negative otherwise.

Immunofluorescence. Cell lines from adherent cultures were digested using $0.25 \%$ trypsin with EDTA at $37^{\circ} \mathrm{C}$ for $8 \mathrm{~min}$ and centrifuged at $180 \mathrm{xg}$ for $4 \mathrm{~min}$ at room temperature. The cell pellet was resuspended in complete DMEM-high glucose (10\% FBS, $1 \%$ penicillin and $1 \%$ streptomycin). Following the preparation of 6-well plates with coverslips, cell suspensions were added to each well $\left(3 \times 10^{5} /\right.$ well $)$. Cells were cultured at $37^{\circ} \mathrm{C}$ in $5 \% \mathrm{CO}_{2}$ for $48 \mathrm{~h}$, washed with PBS and fixed with $4 \%$ paraformaldehyde for $15 \mathrm{~min}$ at room temperature. The cells were subsequently washed with $1 \%$ PBS with Triton-100 to penetrate the cell membrane. Following incubation with $10 \%$ donkey serum (cat. no. 017-000-121; Jackson ImmunoResearch Laboratories, Inc.) at room temperature for $1 \mathrm{~h}$, cells were incubated with primary antibodies against Notch1, Notch2, Notch3, and Notch4 and their ligands Jagged1, Jagged2, DLL1, DLL3 and DLL4 (Table II) at $4^{\circ} \mathrm{C}$ overnight. The signals were generated following incubation with Alexa Fluor 594-conjugated donkey anti-rabbit immunoglobulin G (IgG) secondary antibodies, (dilution, 1:1,000; cat. no. R37119; Invitrogen ${ }^{\mathrm{TM}}$; Thermo Fisher Scientific, Inc., Waltham, MA, USA) at room temperature for $1 \mathrm{~h}$. Nuclear staining was performed with DAPI (Sigma-Aldrich; Merck KGaA, Darmstadt, Germany) for $5 \mathrm{~min}$ at room temperature. Stained coverslips were visualized using a laser scanning confocal microscope (Olympus Soft Imaging Solutions GmbH, Münster, Germany) at magnification $x 40$ and $x 100$. The negative control group was incubated with PBS instead of the primary antibodies.

Western blot analysis. The PDAC cell lines HPAC and PANC-1 were cultured in culture flasks and collected when they became confluent. Cells were subsequently homogenized in a radioimmunoprecipitation buffer for protein extraction $[50 \mathrm{mM}$ Tris (pH 7.4), $150 \mathrm{mM} \mathrm{NaCl}, 1 \%$ Triton $\mathrm{X}-100,0.1 \%$ sodium dodecyl sulfate, $1 \%$ sodium deoxycholate, $10 \mu \mathrm{l} / \mathrm{ml}$ protease inhibitor cocktail and $1 \mathrm{mM}$ phenylmethylsulfonyl fluoride; cat. no. P0013B; Beyotime Institute of Biotechnology]. The protein samples were separated by either 8 or $10 \%$ SDS-PAGE and subsequently transferred onto nitrocellulose membranes (Merck KGaA). Following 3 washes for $10 \mathrm{~min} /$ wash with $20 \mathrm{mM}$ Tris- $\mathrm{Cl}$ (pH 7.5), $0.15 \mathrm{M} \mathrm{NaCl}$ and $0.05 \%$ Tween-20 
Table I. Patient information.

\begin{tabular}{|c|c|c|c|c|c|}
\hline No. & Age & Sex & TNM & Grade & Stage \\
\hline 1 & 77 & $\mathrm{~F}$ & T2N0M0 & 1 & I \\
\hline 2 & 46 & $\mathrm{~F}$ & T3N0M0 & 1 & II \\
\hline 3 & 56 & $\mathrm{~F}$ & T2NOMO & 1 & I \\
\hline 4 & 47 & $\mathrm{~F}$ & T3N0M0 & 1 & II \\
\hline 5 & 64 & $\mathrm{~F}$ & T2N0M0 & 1 & I \\
\hline 6 & 77 & $\mathrm{M}$ & T2N0M0 & 1 & I \\
\hline 7 & 67 & $\mathrm{~F}$ & T3N0M0 & 1 & III \\
\hline 8 & 50 & M & T3N0M0 & 1 & II \\
\hline 9 & 48 & $\mathrm{~F}$ & T3N0M0 & 1 & II \\
\hline 10 & 77 & M & T3N0M0 & 1 & II \\
\hline 11 & 65 & M & T2N0M0 & 1 & I \\
\hline 12 & 47 & $\mathrm{M}$ & T3N0M0 & 1 & II \\
\hline 13 & 61 & M & T2N0M0 & 1 & $\mathrm{I}$ \\
\hline 14 & 65 & $\mathrm{M}$ & T3N0M0 & 2 & II \\
\hline 15 & 57 & $\mathrm{M}$ & T3N0M0 & 2 & II \\
\hline 16 & 38 & $\mathrm{M}$ & T3N0M1 & 2 & IV \\
\hline 17 & 39 & $\mathrm{M}$ & T3N0M0 & 2 & II \\
\hline 18 & 31 & $\mathrm{M}$ & T3N0M0 & 2 & II \\
\hline 19 & 42 & M & T3N0M0 & 1 & II \\
\hline 20 & 44 & M & T3N0M0 & 2 & II \\
\hline 21 & 57 & M & T3N0M0 & 2 & II \\
\hline 22 & 59 & $\mathrm{M}$ & T3N0M0 & 2 & II \\
\hline 23 & 75 & $\mathrm{~F}$ & T3N0M1 & 2 & IV \\
\hline 24 & 52 & $\mathrm{M}$ & T1N0M0 & 2 & I \\
\hline
\end{tabular}

TNM, tumor, node and metastasis; M, male; F, female. T1, tumor invading the submucosa; $\mathrm{T} 2$, tumor invading the muscularis propria; $\mathrm{T} 3$, tumor invading through the muscularis propria into the subserosa or into the non-peritonealized pericolic or perirectal tissues; N0, no regional lymph node metastasis; $\mathrm{N} 1$, metastasis in 1-3 regional lymph nodes; M0, no distant metastasis; M1, distant metastasis.

Table II. Antibodies used within the study.

\begin{tabular}{lclllll}
\hline & & \multicolumn{2}{c}{ Dilution } & & \\
\cline { 3 - 4 } Antigen & Host species & IHC & WB & Supplier & Cat. no. \\
\hline Notch1 & Rabbit & $1: 50$ & $1: 500$ & SC & sc-6014R \\
Notch2 & Rabbit & $1: 500$ & $1: 2,000$ & LS & LS-B399 \\
Notch3 & Rabbit & $1: 50$ & $1: 500$ & & SC & sc-5593 \\
Notch4 & Rabbit & $1: 50$ & $1: 500$ & & SC & sc-5594 \\
Jagged1 & Rabbit & $1: 50$ & $1: 500$ & & SC & sc-8303 \\
Jagged2 & Rabbit & $1: 50$ & $1: 500$ & & SC & sc-5604 \\
DLL1 & Rabbit & $1: 50$ & $1: 500$ & & Ab & ab76655 \\
DLL3 & Rabbit & $1: 100$ & $1: 1,000$ & CS & $2483 s$ \\
DLL4 & Rabbit & $1: 50$ & $1: 1,000$ & BR & HP1274 \\
\hline
\end{tabular}

SC, Santa Cruz Biotechnology, Inc., Dallas, TX, USA; Ab, Abcam, Cambridge, MA, USA; CS, Cell Signaling Technology, Inc., Danvers, MA, USA; LS, LifeSpan Biosciences, Inc., Seattle, WA, USA; BR, Bio-Rad Laboratories, Inc., Hercules, CA, USA; IHC, immunohistochemistry; WB, western blot analysis.

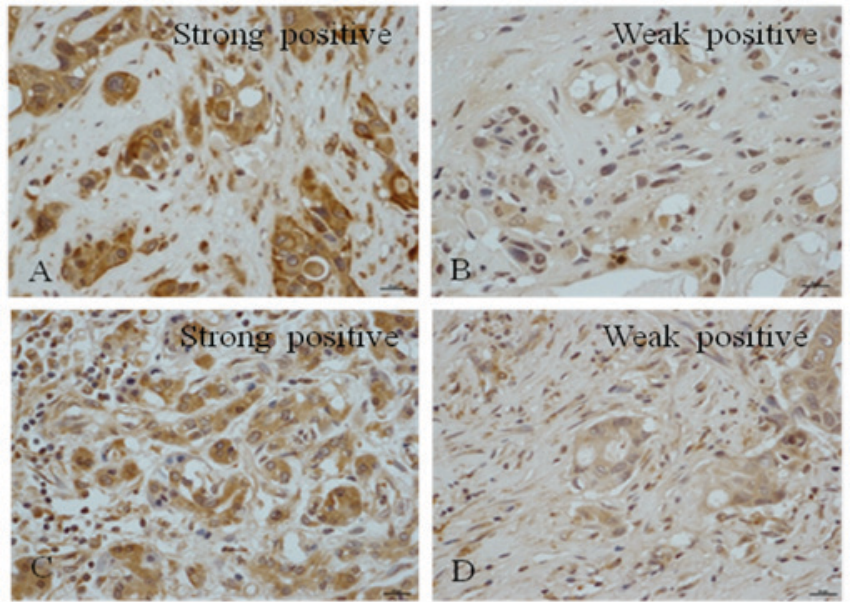

Figure 1. Representative examples of immunohistochemistical staining for Notch receptors in PDAC tissues, indicating that Notch1 and 3 expression levels are increased whereas Notch 2 and 4 levels are decreased in PDAC tissue. Rabbit polyclonal anti-Notch antibodies were used to determine the expression of (A) Notch1, (B) Notch2, (C) Notch3 and (D) Notch4 in PDAC tissues. Scale bar $=20 \mu \mathrm{m}$; magnification $\times 40$. PDAC, pancreatic ductal adenocarcinoma

(TBST), cells were blocked with 5\% skimmed milk in TBST for $1 \mathrm{~h}$ at room temperature. Membranes were then incubated overnight with primary antibodies at $4^{\circ} \mathrm{C}$ (Table II). The membranes were subsequently incubated with secondary goat anti-rabbit IgG HRP-conjugated antibodies (1:1,000; cat. no. 111-625-144; LI-COR Biosciences, Lincoln, NE, USA) for $1 \mathrm{~h}$ at room temperature. Protein bands were visualized using a chemiluminescence detection system (Odyssey ${ }^{\circledR}$ two-color infrared fluorescence imaging system; LI-COR Biosciences). Protein levels were normalized to GAPDH $(1: 10,000$; cat. no. G9545; Sigma-Aldrich; Merck KGaA) levels and quantified using ImageJ software version 1.43b (National Institutes of Health, Bethesda, MD, USA).

Statistical analysis. Data are expressed as the mean \pm standard error of the mean. Statistical analysis was performed using SPSS software, version 19.0 (IBM Corp., Armonk, NY, USA). Pearson's correlation co-efficient was used to identify whether there were correlations between the expression of Notch receptors and their ligands. $\mathrm{P}<0.05$ was considered to indicate a statistically significant difference.

\section{Results}

Positive rate and intensity of Notch receptor expression. Rabbit polyclonal anti-Notch1-4 antibodies were used to detect the expression of Notch1-4 in human PDAC tissues (Fig. 1). It was observed that Notch1 was expressed in all PDAC samples; $91.7 \%$ of the tissues exhibited strong positive staining and $8.3 \%$ demonstrated weak positive staining; none of the samples were negative for Notch1 (Table III). A total of $41.7 \%$ of the samples were negative for Notch2, 37.5\% exhibited weak positivity and $20.8 \%$ of the samples exhibited positive nuclear staining (Table III). It was observed that $41.7 \%$ of the samples had positive staining for Notch3, among which $12.5 \%$ were strongly positive (Table III). Among the samples, $45.8 \%$ were weakly positive for Notch 3 and $12.5 \%$ 
Table III. Positive rate and intensity of Notch receptors and ligands expression.

\begin{tabular}{lrrrrc}
\hline Antigen & $0(\%)$ & $1(\%)$ & $2(\%)$ & $3(\%)$ & Total positive rate $(\%)$ \\
\hline Notch1 & 0.0 & 8.3 & 25.0 & 66.7 & 91.7 \\
Notch2 & 41.7 & 37.5 & 20.8 & 0.0 & 20.8 \\
Notch3 & 12.5 & 45.8 & 29.2 & 12.5 & $41.7^{\mathrm{a}}$ \\
Notch4 & 41.7 & 20.8 & 33.3 & 4.2 & $37.5^{\mathrm{b}}$ \\
JAGGED1 & 62.5 & 20.9 & 8.3 & 8.3 & $16.6^{\mathrm{b}}$ \\
JAGGED2 & 45.8 & 16.7 & 37.5 & 0.0 & $37.5^{\mathrm{b}}$ \\
DLL1 & 0.0 & 20.8 & 25.0 & 54.2 & $79.2^{\mathrm{a}}$ \\
DLL3 & 25.0 & 25.0 & 29.2 & 20.8 & $50^{\mathrm{a}}$ \\
DLL4 & 8.3 & 29.2 & 12.5 & 50.0 & $62.5^{\mathrm{a}}$
\end{tabular}

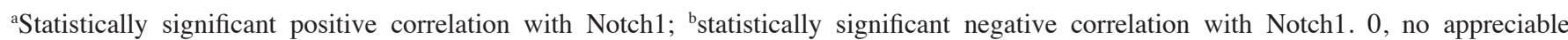
staining-negative; 1 , barely detectable staining-weak positive; 2 , readily identifiable brown staining-positive; 3 , dark brown staining-strong positive. DLL, $\delta$-like ligand.
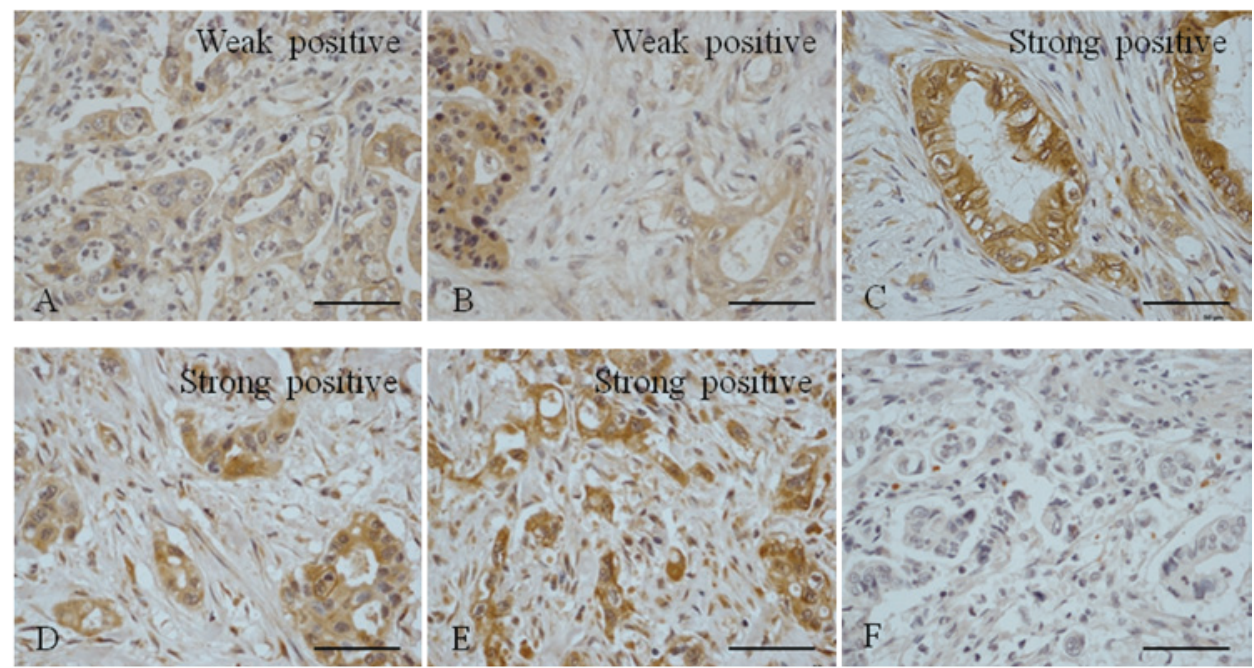

Figure 2. Representative examples of immunohistochemistry staining for Notch ligands in pancreatic ductal adenocarcinoma tissues, indicating that DLL1, DLL3 and DLL4 levels are increased whereas Jagged 1 and 2 levels remain low in PDAC. Rabbit polyclonal antibodies were used to measure the expression of (A) Jagged1, (B) Jagged2, (C) DLL1, (D) DLL3 and (E) DLL4 in PDAC tissues. (F) negative control. Scale bar=50 $\mu$ m. PDAC, pancreatic ductal adenocarcinoma; DLL, $\delta$-like ligand.

were negative (Table III). A total of $41.7 \%$ of the samples were negative for Notch4, $20.8 \%$ were weakly positive, $37.5 \%$ exhibited positive staining and $4.2 \%$ exhibited strong positive expression (Table III).

Positive rate and intensity of Notch ligand expression. Polyclonal Jagged1 and 2 antibodies were used to detect the expression of Jagged1 and 2 in PDAC tissues (Fig. 2). It was observed that $62.5 \%$ of the samples were negative for Jagged 1 expression and $20.9 \%$ were weakly positive; $16.6 \%$ of samples were positive for Jagged 1 but only $8.3 \%$ exhibited strong positive expression (Table II). A total of $45.8 \%$ of the samples were negative for Jagged 2 expression, $16.7 \%$ were weakly positive and $37.5 \%$ exhibited positive staining.

It was observed that only $20.8 \%$ of the PDAC tissue samples exhibited weak positive staining for DLL1; $79.2 \%$ were positive for DLL1 and 54.2\% exhibited strong expression (Table III). None of the samples were negative for DLL1. A total of $25 \%$ of the PDAC tissue samples were negative for DLL3 expression, $25 \%$ were weakly positive and $50 \%$ were positive, with $20.8 \%$ exhibiting strong positive expression (Table II). The results revealed that $8.3 \%$ of the PDAC tissue samples were negative for DLL4, 29.2\% were weakly positive and $62.5 \%$ were positive, with $50 \%$ of the samples exhibiting strong positive expression (Table III). These results were similar to the results obtained regarding DLL1 expression.

Correlation between the expression of Notch receptors and their ligands. As demonstrated in Figs. 1 and 2 and Table III, the expression of Notch receptors and their ligands were examined. It was observed that the majority of PDAC tissue samples (91.7\%) exhibited high Notch1 expression. A total of $41.7 \%$ of samples exhibited positive Notch3 expression. Among the ligands, the majority of PDAC tissues $(79.2 \%)$ stained positive for DLL1; $62.5 \%$ stained positive for DLL4 and 50\% stained positive for DLL3 (Table II). A significant positive correlation 


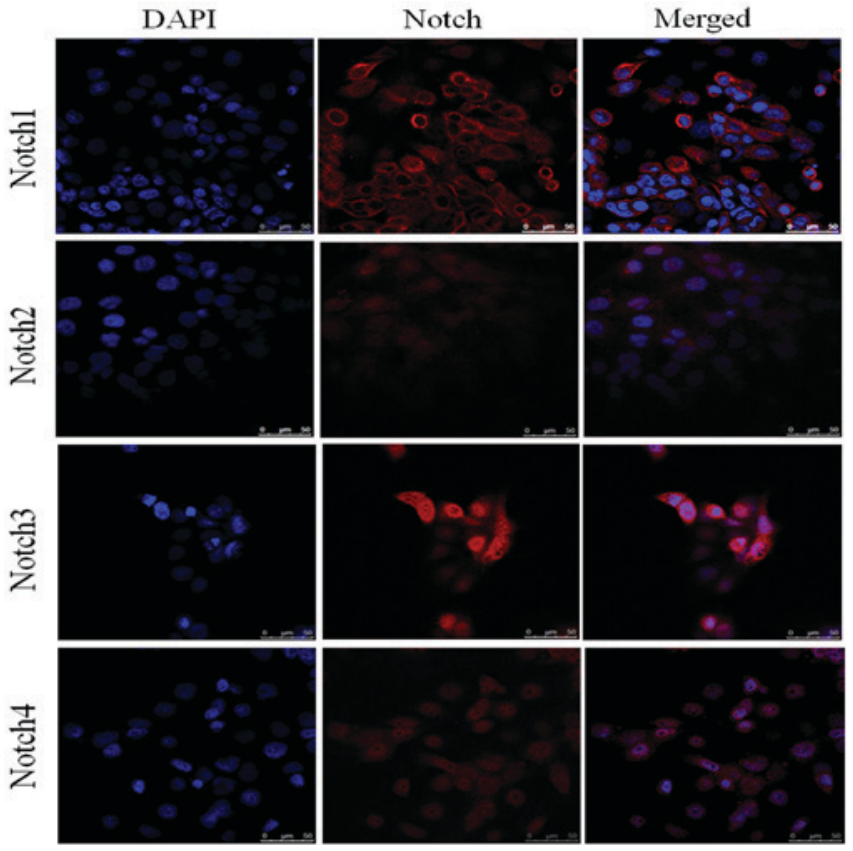

Figure 3. Representative examples of immunofluorescent staining for Notch receptors in HPAC pancreatic cancer cells. DAPI staining is indicated in blue and Notch staining is indicated in red. Scale bar=50 $\mu \mathrm{m}$. HPAC, human pancreatic adenocarcinoma.

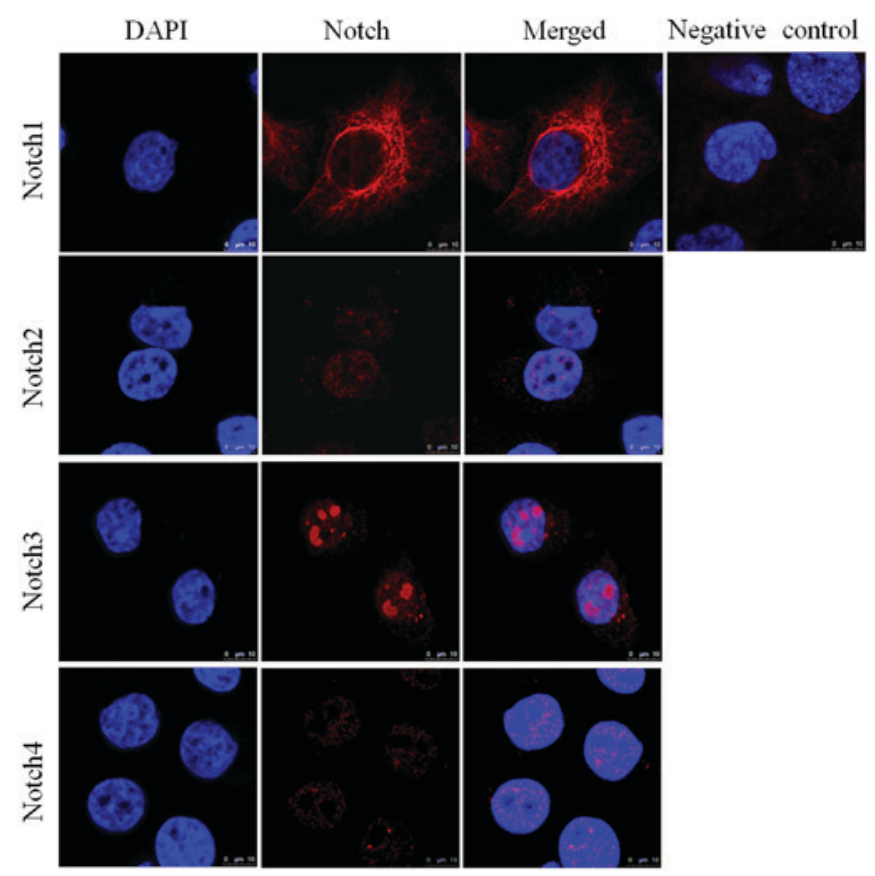

Figure 4. Representative examples of immunofluorescent staining for Notch receptors in PANC-1 pancreatic cancer cells. DAPI staining is indicated in blue and Notch is indicated in red. PBS was used for the negative control Scale bar $=10 \mu \mathrm{m}$. PDAC, pancreatic ductal adenocarcinoma.

between Notch1 and DLL1 was observed. Notch1 also exhibited a positive correlation with DLL4 and DLL3 ( $\mathrm{r}=0.7 ; \mathrm{P}=0.0020)$. However, Notch1 was negatively correlated with Notch4, Jagged1 and Jagged2. Notch 2 expression was positively correlated with Notch4, Jagged1 and Jagged2 ( $r=0.5 ; \mathrm{P}=0.0087)$.

Expression of Notch receptors in pancreatic cancer cell lines assessed by immunofluorescence analysis. Immunofluorescence analysis of HPAC (Fig. 3) and PANC-1 (Fig. 4) cell lines assessed the expression of Notch1, Notch2, Notch3 and Notch4 in each of these cell lines. DAPI (blue) was used to stain the nucleus. Notch1 exhibited positive expression in the cytoplasm and around the nucleus, whereas Notch3 had clear nuclear localization (Fig. 4). The expression of Notch 2 and Notch 4 was notably lower in each of the cell lines compared with Notch1 and Notch3. The elevated expression of Notch1 and Notch3 in HPAC and PANC-1 cell lines was in accordance with their pattern of expression in tissue samples from patients with PDAC. Similarly, the lower expression of Notch2 and Notch4 in the HPAC and PANC-1 cell lines was consistent with their lower expression in PDAC tissue samples.

Expression of Notch receptors in pancreatic cancer cell lines determined by western blot analysis. The expression of the Notch receptors in the pancreatic HPAC and PANC-1 cell lines was assessed (Fig. 5). The results revealed that the expression of Notch1 was notably increased in HPAC cells compared with PANC-1 cells, while Notch3 was highly expressed in the two cell lines. The expression of Notch 2 and Notch 4 was markedly lower than Notch1 and Notch3 in the two cell lines. The protein expression of the Notch ligands in HPAC and PANC-1 cells was also measured (Fig. 6). Levels of DLL1, DLL3 and DLL4 expression were higher than those of Jagged 2 in each of the cell lines. The expression of Jagged1 was notably higher in HPAC cells compared with PANC-1 cells. The specificity of the antibodies was confirmed in HeLa and 293T cell lines (data not shown).

\section{Discussion}

In the present study, immunohistochemistry was performed to evaluate the expression of proteins in the Notch signaling pathway, including various receptors and ligands associated with PDAC. To the best of our knowledge, the current study is the first to evaluate the expression of Notch signaling pathway components and investigate the correlations among them.

The Notch gene was first identified in Drosophila in 1917 (19) and Notch1 was revealed to be associated with T-cell acute lymphoblastic leukemia in 1991 (20). The roles of the Notch signaling pathway in embryonic development $(21,22)$, adult differentiation $(23,24)$, and the development tumors (10-13) have been previously studied and positively confirmed. In eukaryotes, the Notch signaling pathway is highly conserved and regulates cell proliferation, differentiation and apoptosis through interactions between adjacent cells (25-27).

Notch signaling serves a central role in tumors and during the embryonic development of the pancreas, in which it controls cellular differentiation (26). However, the expression and functions of each of the Notch signaling pathway components differ in tumor development, including in PDAC. Miyamoto et al (14) revealed that the expression of the Notch1, Notch2, Notch3 and Notch4 receptors and their ligand Jagged1 was upregulated in resected pancreatic cancer samples. The expression of the Notch signaling target transcription factor Hes1 (Hes1) was also upregulated in pancreatic cancer cells (14). However, Vo et al (16) reported that among the Notch family members, Notch3 was primarily overexpressed in pancreatic cancer, followed by Notch4 and 

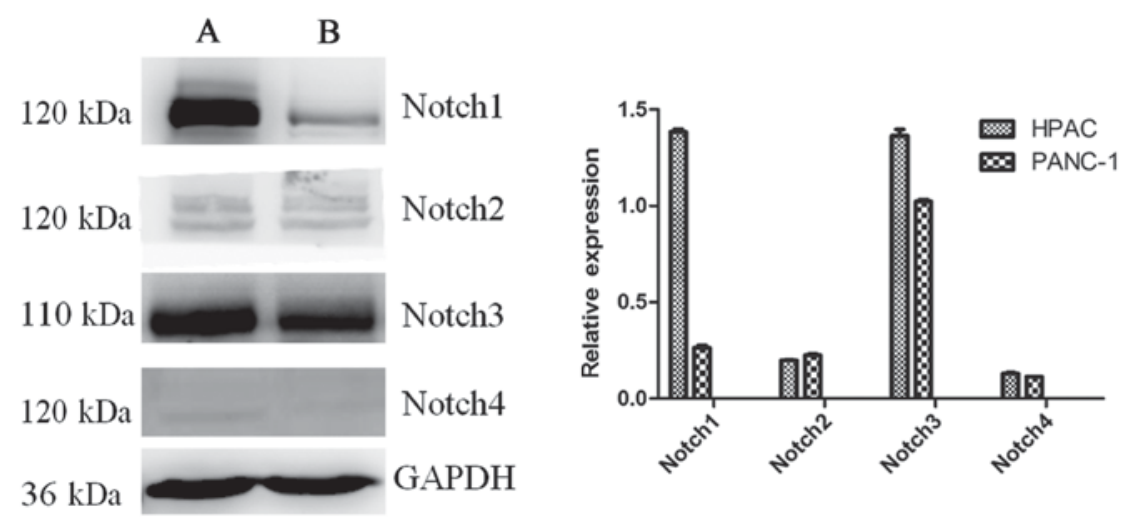

Figure 5. Western blot analysis measuring the expression of Notch receptors in pancreatic cancer cells. (A) HPAC cells; (B) PANC-1 cells. HPAC, human pancreatic adenocarcinoma; PDAC, pancreatic ductal adenocarcinoma.

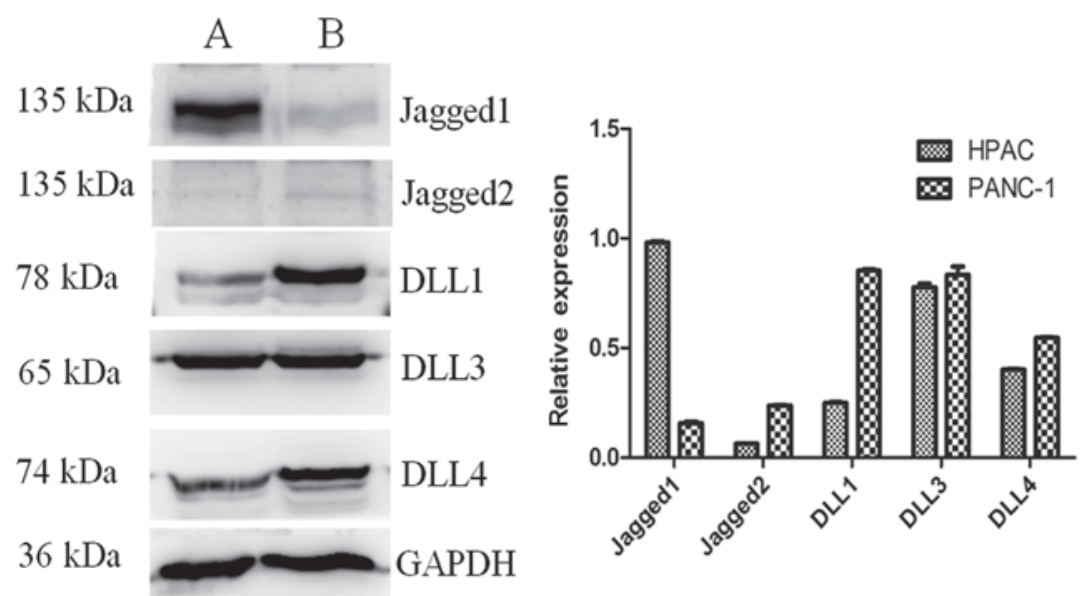

Figure 6. Western blot analysis measuring the expression of Notch ligands in pancreatic cancer cells. (A) HPAC cells; (B) PANC-1 cells. HPAC, human pancreatic adenocarcinoma; PDAC, pancreatic ductal adenocarcinoma; DLL, $\delta$-like ligand.

Jagged1, whereas Notch1 was not expressed in malignant cells. Another previous study demonstrated that Notch 3 was significantly overexpressed in the cytoplasm and nucleus in $43.5 \%$ of pancreatic adenocarcinoma tumors (28). Mann et al (29) demonstrated that the Notch signaling pathway components Notch1, Notch3, Notch4, Hes1 and hairy/enhancer-of-split related with YRPW motif protein 1 were significantly elevated in pancreatic adenocarcinoma. Therefore, it remains unclear how the expression of various Notch proteins changes during the progression of cancer, and to the best of our knowledge a complete investigation examining the expression of all Notch receptors and their ligands in PDAC has not yet been conducted.

The elevated expression of Notch1 in pancreatic cancer leads to the accumulation of undifferentiated precursor cells (30), whereas the downregulation of Notch1 decreases cyclin D1 and B-cell lymphoma 2 expression, which increases the apoptosis of pancreatic cancer cells (31). It has been demonstrated that inhibiting the Notch signaling pathway using Notch1 small interfering RNA triggers apoptosis in the pancreatic cancer cell lines BxPC-3, MIAPaCa-2 and PANC-1 (32). Blocking Notch2/3 inhibits tumor growth and tumor-initiating cells (33) and the inhibition of Notch1 and Notch4 expression inhibits tumor growth $(34,35)$. Mazur et al $(11)$ demonstrated that Notch2 is a central regulator of pancreatic intraepithelial neoplasia progression and malignant transformation. Notch1 has also been reported to function as a tumor suppressor gene in PDAC (18). A number of studies have reported conflicting results and the role of Notch signaling in PDAC remains highly controversial. In addition, there is little information available concerning the expression pattern of Notch receptors and their ligands in PDAC.

Therefore, the present study was conducted to estimate the expression and potential pathological significance of all Notch receptors and their ligands in human PDAC. In the present study, Notch1 exhibited increased expression in PDAC tissues, in which it may serve a role in the development of pancreatic cancer development by acting as an oncogene. Notch 3 was also highly expressed, suggesting that it serves a similar role to Notch1 in PDAC. The DLL1, DLL3 and DLL4 ligands were upregulated. By contrast, levels of Notch2 and Notch 4 were decreased in PDAC tissues. In the cohort of patients assessed in the current study, the expression of the ligands Jagged1 and Jagged2 were also decreased compared with DLL1, DLL3 and DLL4.

To determine the expression and potential functions of these molecules, HPAC and PANC-1 pancreatic cancer cell lines were selected and immunofluorescence staining and western blot analysis was performed. The results of immunofluorescence staining revealed that Notch1 was expressed in the 
cytoplasm and around the nucleus. The expression of Notch3 was also positive, however, it was localized in the nucleus. Levels of Notch2 and Notch4 were decreased compared with Notch1 and Notch3. Therefore, the expression of Notch1 and Notch 3 in the pancreatic cancer cell lines corresponded with their expression in PDAC cancer tissues, confirming that they are highly expressed in PDAC. The expression of Notch 2 and Notch4 in the cancer cell lines was also consistent with their expression in cancer tissues; Notch2 and Notch4 expression were decreased compared with Notch1 and Notch3. Western blot analysis also revealed notably elevated expression of Notch1 and Notch3 compared with Notch2 and Notch4 in the pancreatic cancer cell lines HPAC and PANC-1. The Notch ligands DLL1, DLL3 and DLL4 exhibited markedly higher expression than that of Jagged2.

In the present study, a positive correlation was observed between the expression of Notch1 and Notch3, and between Notch1 and the ligands DLL1, DLL3 and DLL4. The results of the western blot analysis were consistent with those of immunohistochemistry, suggesting that the Notch1 and Notch3 pathways may be initiated by DLL1, DLL3 or DLL4. However, they do not seem to be initiated by Jagged2.

Due to the limited number of patients recruited and the lack of normal controls in the present study, these results may not be representative of the entire population. Future studies should be conducted to investigate a greater number of samples to confirm the results of the present study. Understanding the molecular characteristics of tumors may provide an important basis for the clinical diagnosis and treatment of patients with pancreatic cancer. The present study suggested that Notch1 and Notch3 may be potential targets for treatments against PC, and may provide the basis for a novel method of treatment and diagnosis of PC in the future.

\section{Acknowledgements}

Not applicable.

\section{Funding}

The study was supported by the National Natural Science Foundation of China (grant no. 81372156) and the Doctor Scientific Research Foundation of Xinxiang Medical University (grant no. XXBSKYZZ201821).

\section{Availability of data and materials}

All data generated or analyzed during this study are included in this published article.

\section{Authors' contributions}

YZ and HS conceived and designed the experiments. HS, YW and HL conducted the experiments.

\section{Ethics approval and consent to participate}

The present study was approved by the Ethics Committee of the Affiliated Center Hospital of Xinxiang Medical University and written informed consent was obtained from all patients prior to their inclusion within the study.

\section{Consent for publication}

All patients provided written consent for the publication of their data.

\section{Competing interests}

The authors declare that they have no competing interests.

\section{References}

1. Ryan DP, Hong TS and Bardeesy N: Pancreatic adenocarcinoma. N Engl J Med 371: 1039-1049, 2014.

2. Jemal A, Bray F, Center MM, Ferlay J, Ward E and Forman D: Global cancer statistics. CA Cancer J Clin 61: 69-90, 2011.

3. Siegel RL, Miller KD and Jemal A: Cancer statistics, 2016. CA Cancer J Clin 66: 7-30, 2016.

4. Bliss LA, Witkowski ER, Yang CJ and Tseng JF: Outcomes in operative management of pancreatic cancer. J Surg Oncol 110: 592-598, 2014

5. Ranganathan P, Weaver KL and Capobianco AJ: Notch signalling in solid tumours: A little bit of everything but not all the time. Nat Rev Cancer 11: 338-351, 2011.

6. Apelqvist A, Li H, Sommer L, Beatus P, Anderson DJ, Honjo T, de Angelis MH, Lendahl $\mathrm{U}$ and Edlund $\mathrm{H}$ : Notch signalling controls pancreatic cell differentiation. Nature 400: 877-881, 1999.

7. Artavanis-Tsakonas S, Rand MD and Lake RJ: Notch signaling: Cell fate control and signal integration in development. Science 284: 770-776, 1999.

8. Espinoza I and Miele L: Deadly crosstalk: Notch signaling at the intersection of EMT and cancer stem cells. Cancer Lett 341: 41-45, 2013

9. Wang J, Han F, Wu J, Lee SW, Chan CH, Wu CY, Yang WL, Gao Y, Zhang X, Jeong YS, et al: The role of Skp2 in hematopoietic stem cell quiescence, pool size, and self-renewal. Blood 118: 5429-5438, 2011.

10. Oishi H, Sunamura M, Egawa S, Motoi F, Unno M, Furukawa T, Habib NA and Yagita H: Blockade of delta-like ligand 4 signaling inhibits both growth and angiogenesis of pancreatic cancer. Pancreas 39: 897-903, 2010.

11. Mazur PK, Einwächter H, Lee M, Sipos B, Nakhai H, Rad R, Zimber-Strobl U, Strobl LJ, Radtke F, Klöppel G, et al: Notch2 is required for progression of pancreatic intraepithelial neoplasia and development of pancreatic ductal adenocarcinoma. Proc Natl Acad Sci USA 107: 13438-13443, 2010.

12. Mizuma M, Rasheed ZA, Yabuuchi S, Omura N, Campbell NR, de Wilde RF, De Oliveira E, Zhang Q, Puig O, Matsui W, et al: The gamma secretase inhibitor MRK-003 attenuates pancreatic cancer growth in preclinical models. Mol Cancer Ther 11: 1999-2009, 2012

13. Hu Y, Su H, Li X, Guo G, Cheng L, Qin R, Qing G and Liu H: The NOTCH ligand JAGGED2 promotes pancreatic cancer metastasis independent of NOTCH signaling activation. Mol Cancer Ther 14: 289-297, 2015.

14. Miyamoto Y, Maitra A, Ghosh B, Zechner U, Argani P, Iacobuzio-Donahue CA, Sriuranpong V, Iso T, Meszoely IM, Wolfe MS, et al: Notch mediates TGF alpha-induced changes in epithelial differentiation during pancreatic tumorigenesis. Cancer Cell 3: 565-576, 2003.

15. Thomas MM, Zhang Y, Mathew E, Kane KT, Maillard I and Pasca di Magliano M: Epithelial Notch signaling is a limiting step for pancreatic carcinogenesis. BMC Cancer 14: 862, 2014.

16. Vo K, Amarasinghe B, Washington K, Gonzalez A, Berlin J and Dang TP: Targeting notch pathway enhances rapamycin antitumor activity in pancreas cancers through PTEN phosphorylation. Mol Cancer 10: 138, 2011.

17. Gao J, Long B and Wang Z: Role of Notch signaling pathway in pancreatic cancer. Am J Cancer Res 7: 173-186, 2017.

18. Hanlon L, Avila JL, Demarest RM, Troutman S, Allen M, Ratti F, Rustgi AK, Stanger BZ, Radtke F, Adsay V, et al: Notch1 functions as a tumor suppressor in a model of K-ras-induced pancreatic ductal adenocarcinoma. Cancer Res 70: 4280-4286, 2010. 
19. Morgan MM and Mahowald AP: Multiple signaling pathways establish both the individuation and the polarity of the oocyte follicle in Drosophila. Arch Insect Biochem Physiol 33: 211-230, 1996.

20. Ellisen LW, Bird J, West DC, Soreng AL, Reynolds TC, Smith SD and Sklar J: TAN-1, the human homolog of the Drosophila notch gene, is broken by chromosomal translocations in T lymphoblastic neoplasms. Cell 66: 649-661, 1991.

21. Piccirilli D, Baldini E, Massimiani M, Camaioni A, Salustri A, Bernardini R, Centanni M, Ulisse S, Moretti C and Campagnolo L: Thyroid hormone regulates protease expression and activation of Notch signaling in implantation and embryo development. J Endocrinol 236: 1-12, 2018.

22. Hussain M, Xu C, Ahmad M, Yang Y, Lu M, Wu X, Tang L and Wu X: Notch signaling: Linking embryonic lung development and asthmatic airway remodeling. Mol Pharmacol 92: 676-693, 2017.

23. Li J, Chen SY, Zhao XY, Zhang MC and Xie HT: Rat limbal niche cells prevent epithelial stem/progenitor cells from differentiation and proliferation by inhibiting Notch signaling pathway in vitro. Invest Ophthalmol Vis Sci 58: 2968-2976, 2017.

24. Yu Z, Zou Y, Fan J, Li C and Ma L: Notch1 is associated with the differentiation of human bone marrow-derived mesenchymal stem cells to cardiomyocytes. Mol Med Rep 14: 5065-5071, 2016.

25. Williams E, Villar-Prados A, Bowser J, Broaddus R and Gladden AB: Loss of polarity alters proliferation and differentiation in low-grade endometrial cancers by disrupting Notch signaling. PLoS One 12: e0189081, 2017.

26. Irles P, Elshaer N and Piulachs MD: The Notch pathway regulates both the proliferation and differentiation of follicular cells in the panoistic ovary of Blattella germanica. Open Biol 6: 15019, 2016

27. Wang L, Song G, Liu M, Chen B, Chen Y, Shen Y, Zhu J and Zhou X: MicroRNA-375 overexpression influences P19 cell proliferation, apoptosis and differentiation through the Notch signaling pathway. Int J Mol Med 37: 47-55, 2016.

28. Doucas H, Mann CD, Sutton CD, Garcea G, Neal CP, Berry DP and Manson MM: Expression of nuclear Notch3 in pancreatic adenocarcinomas is associated with adverse clinical features and correlates with the expression of STAT3 and phosphorylated Akt. J Surg Oncol 97: 63-68, 2008.
29. Mann CD, Bastianpillai C, Neal CP, Masood MM, Jones DJ, Teichert F, Singh R, Karpova E, Berry DP and Manson MM: Notch3 and HEY-1 as prognostic biomarkers in pancreatic adenocarcinoma. PLoS One 7: e51119, 2012.

30. Sjolund J, Manetopoulos C, Stockhausen MT and Axelson H: The Notch pathway in cancer: Differentiation gone awry. Eur J Cancer 41: 2620-2629, 2005.

31. Wang Z, Zhang Y, Li Y, Banerjee S, Liao J and Sarkar FH Down-regulation of Notch-1 contributes to cell growth inhibition and apoptosis in pancreatic cancer cells. Mol Cancer Ther 5: 483-493, 2006.

32. Du X, Wang YH, Wang ZQ, Cheng Z, Li Y, Hu JK, Chen ZX and Zhou ZG: Down-regulation of Notch1 by small interfering RNA enhances chemosensitivity to gemcitabine in pancreatic cancer cells through activating apoptosis activity. Zhejiang Da Xue Xue Bao Yi Xue Ban 43: 313-318, 2014 (In Chinese).

33. Yen WC, Fischer MM, Axelrod F, Bond C, Cain J, Cancilla B, Henner WR, Meisner R, Sato A, Shah J, et al: Targeting Notch signaling with a Notch2/Notch3 antagonist (tarextumab) inhibits tumor growth and decreases tumor-initiating cell frequency. Clin Cancer Res 21: 2084-2095, 2015.

34. Kunnimalaiyaan S, Trevino J, Tsai S, Gamblin TC and Kunnimalaiyaan M: Xanthohumol-mediated suppression of Notch1 signaling is associated with antitumor activity in human pancreatic cancer cells. Mol Cancer Ther 14: 1395-1403, 2015.

35. Xu Y,Zhu F, Xu S and Liu L: Anti-tumor effect of the extract from qingyihuaji formula on pancreatic cancer by down-regulating Notch-4 and Jagged-1. J Tradit Chin Med 35: 77-83, 2015. 